\title{
PROXIMAL FEMUR LOCKING COMPRESSION PLATE IN SUBTROCHANTERIC FRACTURES : A PROSPECTIVE ANALYSIS
}

\section{Orthopaedics}

Dr Kailasnath M.S College,BIHER,No.7, CLC works road, New colony, Chromepet, Chennai 600044 , Tamil Nadu, India.

Dr K Subramanian*

Professor Department of Orthopaedics,SreeBalaji Medical College,BIHER,No.7, CLC works road, New colony, Chromepet, Chennai 600044, Tamil Nadu, India. *Corresponding Author

Associate Professor Department of Orthopaedics,SreeBalaji Medical College , Dr G. Pugazhendhi BIHER,No.7, CLC works road, New colony, Chromepet, Chennai 600044, Tamil Nadu, India.

\section{ABSTRACT}

Introduction:Sub-trochanteric femoral fractures are injuries that mostly affect the elderly population.Ithasa bimodal distribution. In young and healthy individuals, the injury results from high energy trauma, where as in the elder age group, most of the fractures are in osteoporotic bone resulting from a trivial fall. The aim of the surgery is to achieve initial stability and early mobilization of the patients and to avoid complications. The aim of our study is to analyze the outcomes of proximal femoral locking compression plate (PF-LCP) in these fractures.

Materials and Methods:We prospectively analyzed30sub-trochantericfemoral fractures treated with PF-LCP from January 2015 to December 2018. There were 20 females $(66.6 \%)$ and 10 males (33.4\%) with an average age of 61.2 years (range, 36 to 75 years). The sub-trochanteric fractures are classified by Seinshemier's classification. The functional outcome was evaluated by Harris Hip Score and the Parker Palmer Mobility Score at 12 months follow-up.

Results: Among 30 patients, 27 patients (90\%) obtained fracture union without further intervention; 3 patients required additional bone grafting at the end of 3 months. There were no cases of hip screw cutting the femoral head. There was no post-operative mortality in our study. The average Harris Hip Score was 87 (80-93). The assessment by Parker and Palmar Mobility Score was 7.8(range 5-9).

Conclusions:The PF-LCP is a good stable alternative in the treatment of sub-trochanteric femoral fractures. It providesgood to excellent bone healing with limited complications.

\section{KEYWORDS}

\section{INTRODUCTION}

Sub-trochantericfractures account for $10-35 \%$ of all hip fractures and can affect people of all ages. In the young and healthy individuals, the injury results from high energy trauma, where as in the elder age group, most of the fractures are in the osteoporoticbone, resulting from a trivial fall. A sub-trochanteric femur fracture is fracture between the lesser trochanter and a point $5 \mathrm{~cm}$ distal to the lesser trochanter. As conservative methods resulted in higher mortality rates ranging 20$25 \%$ they are now indicated only for elderly person with high medical risk for anesthesia and surgery. Thus, surgery by internal fixation is the ideal choice. The aim of the surgery is to achieve initial stability and early mobilization of the patients and to avoid complications such as deep vein thrombosis, pulmonary embolism etc.Dilemma exists regarding the choice of implant for these fractures. This study was conducted to review the early outcome of cases of PF-LCP use in subtrochanteric fractures in SreeBalaji Medical College and Hospital in Chennai, India.

Proximal Femur Locking Compression Plate (PF-LCP)is an implant that allows angular-stable plate fixation for the treatment of complex comminuted and osteoporotic fractures in different anatomical locations.PF-LCP provides flexibility to achieve plate to bone apposition as well as axial compression or angular stability because of three screw fixation at the fracture site. Unlike conventional compression plates, the screw head locks into the PF-LCP, thereby creating an angular stable construct. PF-LCP can provide a stress shield for the lateral trochanteric wall and prevent lateral migration of proximal fragments. Thus, PF-LCP does not fail at the screw bone interface and provide a strong anchor in osteoporotic bone. The multiple locking screw holes of the PF-LCP provide various options to tackle complex fracture pattern. It functions as an internalized external fixator and minimizes the pressure on the periosteum and encourages biological healing. Locking plates have the advantage of allowing multiple angularstable fixation points into the proximal femur.It leaves a mark by preserving more bone stock after implantation compared with the use of large proximal lag screws .PF-LCP is a limited contact fixed angular, stable construct with high pullout strength. The screw head locks into the PF-LCP and provides stability,it does not fail at the bone screw interface, and also provides strong anchor in osteoporotic fractures . Extramedullaryimplants are helpful minimizing problems such as abductor weakness and superior gluteal nerve injury .The most widely accepted treatment is intramedullary nailing but there are some challenges like placement of the nail, anatomical reduction in reverse oblique fractures and when fracture line extends into the greater trochanter

Nailing is also fraught with complications including femoral shaft fractures, varus deformity and malrotation. The aim of our study is to analyze the outcomes of PF-LCP in fixation of sub-trochanteric femoral fractures in terms of union, functional outcome, postoperative complications and failure rate.

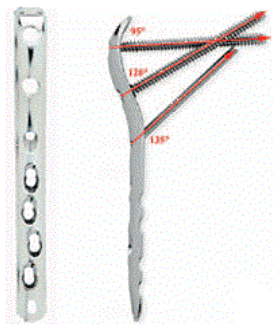

proximal femur locking compression plate.

Case 1.

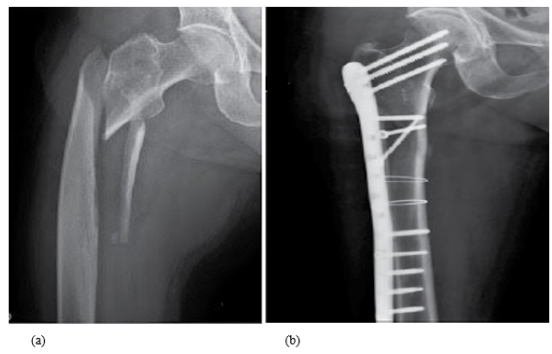


(a)preoperative and (b) postoperative radiographs of 66 year old female (Seinsheimer III) showing sub-trochanteric fracture and PFLCP

Case 2.

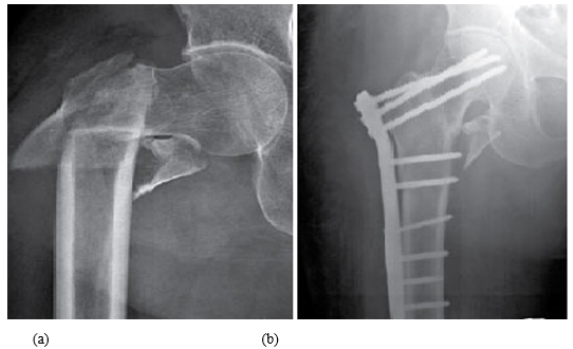

(a)preoperative and (b) postoperative radiographs of a 75 year old female (Seinsheimer V) sub-trochanteric femoral fracture.

\section{MATERIALS AND METHODS}

This study included total of 30 patients admitted to SreeBalaji Medical College and Hospital from January 2015 to December 2018 with subtrochanteric femoral fractures. We prospectively analyzed30subtrochanteric femoral fractures treated with PF-LCP. There were 20 females (66.6\%) and 10 males (33.4\%) with an average age of 61.2 years (range, 36 to 75 years). The sub-trochanteric fractures classified by Seinshemier's classification. The functional outcome was evaluated by Harris Hip Score and the Parker Palmer Mobility Score at 12 months follow-up after surgery.After the surgery patients were followed up evaluated clinically by Harris Hip Score ,radiologically for union at fracture site and implant related complications.

\section{INCLUSION CRITERIA}

Age group of the study: $36-75$ years of both sexes.

Closed fractures

Patients fit for surgery.

\section{EXCLUSION CRITERIA:}

Patients failing anaesthetic fitness.

Polytrauma.

Pathological fractures.

\section{Seinsheimer's classification}

\section{Surgical Technique:}

As soon as the patient is diagnosed withsub-trochantericfracture after necessary clinical and radiological examinationspatient is admitted to the ward after necessary resuscitation and splintage using skin traction. Analgesics and antibiotics were given accordingly. Patients were evaluated for medical comorbidities and cross reference was obtained from other respective departments, wherever necessary. Associated injuries were evaluated and treated simultaneously. All patients were operated on elective basis.Surgery was performed under spinal anaesthesia, supine position on fracture table using straight lateral incision over the greater trochanter extending distally. C-Arm was used to visualize the AP and Lateral views for fracture reduction and implant fixation.A $15 \mathrm{cms}$ vertical incision was taken from the tip of trochanter in distal direction along the shaft of femur. Fascia lata was opened in line with the incision and gluteus medius and vastuslateralis muscles were split in line with the fibers and tip of the trochanter, thereby exposing the proximal femur. Temporary K- wires and Interfragmentary screws were used whenever needed.The fixed-angle wire guides are threaded to the proximal three holes of the plate, and the plate is approximated to the proximal femur. Next, a guide wire was advanced through the most proximal (95degree) hole. The correct path of this wire is approximately one centimetre inferior to the piriformis fossa into the inferior femoral head on the antero-posterior (AP) view, and central in the femoral head on the lateral view. A guide wire is inserted into the next distal (120-degree) hole, and because this is in a different plane than the first hole, the surgeon must visualize its position on the lateral $x$-ray. The third guide wire, in the 135 degree hole, is then placed, which is in the same plane as the first hole and may alternatively be inserted near theend of the procedure without compromising the stability of the construct. All three guide wires should be in subchondral bone of the femur head before inserting the screws which is confirmed by $\mathrm{C}$-arm in the $\mathrm{AP}$ and lateral views. The screw lengths are measured using an indirect device over the guide wires with the wire guides still attached and the appropriate, fully threaded, cannulated screws $(7.3 \mathrm{~mm}$ for the two proximal holes and $5.0 \mathrm{~mm}$ for the third proximal hole) are selected. These cannulated screws are inserted over the guide wires with the guides removed. During distal screws fixation, in sub-trochantric fractures first fracture should be reduced, then fix the non-locking screws in compression mode followed by locking screws. After the completion of the fixation, thorough wash of the wound was given with normal saline. Suction drain was inserted at the entry point and wound closed in layers. Postoperative check X-ray obtained on POD 1. Drain removed on POD 2. Bedsides, knee bending exercises were initiated when pain reduced on third or fourth post-operative day. Patients were reviewed at 12 months follow-up with clinical and radiographic assessment for the progress of fracture healing and complication. The functional outcome was assessed by Harris Hip Score and Parker and Palmer Mobility Score on 12 months follow-up.

\section{RESULTS}

No patients were lost in follow-up and 30 patients were available for the study. Out of 30 cases 4 healed with varus deformity of less than 15 degrees and 6 cases of shortening upto $15 \mathrm{~mm}$. We encountered 3 cases of non-union which went on to heal after bone grafting. All 30 cases had radiological union at 6 to 8 months. There were no intra-operative complications as well as mortality.According to Harris Hip Score the functional outcome were as follows. Excellent (90 point) in 16 cases, Good (80-89 point) in 12cases andFair(70-79) in 2 cases.

\section{DISCUSSION}

The subtrochanteric region of the femur consists primarily of cortical bone. The femoral head and neck are anteverted approximately $10^{\circ}$ to $15^{\circ}$ in relation to the plane of the femoral shaft. The piriformis fossa lies at the base of the neck and is oriented in line with the femoral shaft. The lesser trochanter is postero-medial, and it is the point of insertion for the psoas and iliacus tendons. The femoral shaft has both an anterior and a lateral bow. The major muscles that surround the hip create significant forces that contribute to fracture deformity. The gluteus medius and minimus tendons attach to the greater trochanter and abduct the proximal fragment. The psoas and iliacus attach to the lesser trochanter and flex the proximal fragment. The adductors pull the distal fragment medially. All of these muscles are well vascularized, and this can lead to significant hemorrhage at the time of injury or during surgical approaches.In order to approach the proximal lateral femur, the vastus lateralis must be split or elevated off the intermuscular septum close to the large perforating branches of the profunda femoris artery. Division of these vessels can lead to copious bleeding, making surgical exposure difficult. High failure rates sometimes can be partly attributed to bad technique.

In this study evaluation of 30 patients in terms of functional outcome for sub-trochanteric femoral fractures using PF-LCP by Harris Hip Score. Complications likevarusdeformity, shortening and non-union were seen during study. In our study we had 4 cases of varusdeformity following early weight bearing, but fractures later on went into uneventful union. In our study we used all the three screws in the proximal fragment, which may explain the higher union rate $(90 \%)$. We avoided weight bearing till 8 weeks as we encountered varusdeformity following early weight bearing. In our study the mean time to union was 20 weeks (range, 16 to 24 weeks).

\section{CONCLUSION:}

The PF-LCP is a good stable and feasible alternative in the treatment of sub-trochanteric femoral fractures. It provides good to excellent bone healing with limited complications irrespective of age, sex , number of fragments and bone quality.

\section{ACKNOWLEDGEMENTS:}

Author would like to thank all patients for their cooperation in study.

\section{FUNDING: NO FUNDING SOURCES. CONFLICT OF INTEREST: NONE DECLARED.}

\section{REFERENCES}

1. R GokulNath, SahAnsari .Role of Proximal Femoral Locking Plate in treatment of Subtrochanteric Fractures; Case series. MedPulse International Journal of Orthopedics. July 2017;3(1): 01-07

2. Rajesh Govindasamy, RamkumarGnanasundaram , SaravananKasirajan Jimmy.J.Meleppuram , Kumar Archit. Proximal femur locking compression plate in complex proximal femoral fractures: a retrospective analysis Int J Res Orthop. 2016 Sep;2(3):104-108

Saini P, Kumar R, Shekhawat V, Joshi N, Bansal M, Kumar S.Biological fixation of 
comminuted subtrochanteric fractures with proximal femur locking compression plate.Injury. $2013 \mathrm{Feb}$;44(2):226-31

4. Sun-Jun Hu; Shi-Min Zhang; Guang-RongYu Treatment of femoral subtrochanteric fractures with proximal lateral femur locking plates. ActaOrtopédicaBrasileira vol.20 no.6 São Paulo Nov./Dec. 2012

5. Gunadham U, Jampa J, Suntornsup S, LeewiriyaphunB.The outcome in early cases of treatment of subtrochanteric fractures with proximal femur locking compression plate. Malaysian orthopaedic journal. $2014 \mathrm{Jul} ; 8(2): 22-8$ 